\title{
Plasma density structures at comet 67P/Churyumov-Gerasimenko
}

\author{
I. A. D. Engelhardt, ${ }^{1,2 \star}$ A. I. Eriksson, ${ }^{1 \star}$ G. Stenberg Wieser, ${ }^{3}$ C. Goetz, ${ }^{4}$ M. Rubin, ${ }^{5}$ \\ P. Henri, ${ }^{6}$ H. Nilsson, ${ }^{3}$ E. Odelstad, ${ }^{1,2}$ R. Hajra ${ }^{6}$ and X. Vallières ${ }^{6}$ \\ ${ }^{1}$ Swedish Institute of Space Physics, Box 537, SE-751 21 Uppsala, Sweden \\ ${ }^{2}$ Department of Physics and Astronomy, Uppsala University, Box 516, SE-751 20 Uppsala, Sweden \\ ${ }^{3}$ Swedish Institute of Space Physics, Box 812, SE-981 28 Kiruna, Sweden \\ ${ }^{4}$ Institute for Geophysics and Extraterrestrial Physics, TU Braunschweig, D-38106 Braunschweig, Germany \\ ${ }^{5}$ Space Research and Planetary Sciences, University of Bern, CH-3012 Bern, Switzerland \\ ${ }^{6}$ LPC2E, CNRS, Université d'Orléans, F-45100 Orléans, France
}

Accepted 2018 March 20. Received 2018 March 7; in original form 2018 February 7

\begin{abstract}
We present a Rosetta Plasma Consortium (RPC) case study based on four events in 2015 autumn at various radial distances, phase angles and local times, just after the perihelion of comet 67P/Churyumov-Gerasimenko. Pulse-like (high-amplitude, up to minutes in time) signatures are seen with several RPC instruments in the plasma density (with the LAngmuir Probe, LAP and Mutual Impedance Probe, MIP), ion energy and flux (with the Ion Composition Analyzer, ICA) and the magnetic field intensity (with the magnetometer, MAG). Furthermore, the cometocentric distance relative to the electron exobase is seen to be a good organizing parameter for the measured plasma variations. The closer Rosetta is to this boundary, the more pulses are measured. This is consistent with the pulses being filaments of plasma originating from the diamagnetic cavity boundary, as predicted by simulations.
\end{abstract}

Key words: plasmas - comets: individual: 67P/Churyumov-Gerasimenko.

\section{INTRODUCTION}

The Rosetta mission is the first of its kind. The spacecraft closely followed and orbited comet 67P/Churyumov-Gerasimenko (hereafter 67P) for about 2 years (2014 August to 2016 September). The recorded data cover heliocentric distances between 1.25 and about 4 au (see for example Taylor et al. 2017 for an overview of the mission) as the comet moved along its orbit towards and away from the sun. This was an excellent opportunity to follow the evolution and dynamics of the cometary coma, including its plasma component.

During the whole Rosetta mission, 67P was less active than 1P/Halley when it was visited by Giotto at perihelion in 1986 (de Almeida et al. 2009; Hansen et al. 2016). Collisions of the charged particles with neutral gas molecules are therefore less frequent for 67P. Electron cooling on the neutral gas is thus less efficient, and warm electrons (around 5-10 eV) dominate the electron flux in the inner coma. This can be seen from the spacecraft potential, which typically was at least $5 \mathrm{~V}$ negative for plasma densities above $\sim 100 \mathrm{~cm}^{-3}$ (Odelstad et al. 2015, 2017). Electrons at energies of up to several $100 \mathrm{eV}$ are regularly observed, with tails on the energy distribution up to $10 \mathrm{keV}$ (Broiles et al. 2016). Nevertheless, cooler electrons (below $0.1 \mathrm{eV}$ ) were occasionally picked up by the Rosetta Langmuir probe (Eriksson et al. 2017) and sometimes dominated the plasma density. Because the energy distribution of recently ionized photoelectrons is flat and wide up to tens of electronvolts (Vigren \&

^E-mail: ilka.engelhardt@irfu.se (IADE); Anders.Eriksson@irfu.se (AIE)
Galand 2013), the cold electrons must have undergone collisional cooling in the innermost coma.

The limiting distance outside of which electrons are no longer collisional, and therefore the cooling is inefficient, is known as the electron exobase, also called the electron collisionopause or electron cooling boundary (Mandt et al. 2016; Eriksson et al. 2017; Henri et al. 2017). This is not a sharp boundary but a region of gradual transition, and the exobase distance can be seen as a characteristic scale-length. It is defined as the distance to the comet where the neutral gas density scale-height, $\mathrm{H}$, is equal to the electron mean free path. This is the same definition as is used for the exobase in a planetary atmosphere, where the scale-height, $H$, is that of hydrostatic equilibrium, $H=\mathrm{kT} / \mathrm{mg}$ and $\mathrm{k}$ is the boltzman constant, $\mathrm{T}$ is temperature in Kelvin, $\mathrm{m}$ is mass and $\mathrm{g}$ is gravitational acceleration. However, in the expanding comet atmosphere the neutral gas density decays as $1 / r^{2}$ so the scale-height at distance $r$ is $H \sim r$ (Hansen et al. 2016). It can be noted that while the atmosphere is not spherically symmetric, the expansion is still expected to be radial and the neutral gas density depends on the distance as $1 / r^{2}$ (Tenishev, Combi \& Davidsson 2008).

Inside the exobase, the electrons are assumed to lose most of their energy as a result of collisions with neutrals. The electron pressure inside is therefore low, while the electron pressure outside this boundary remains high. The exobase distance $L_{\mathrm{c}}$ can be expressed in terms of the neutral density $n_{\mathrm{n}}$ at any given cometocentric distance $r$ as (Eriksson et al. 2017)

$L_{\mathrm{c}}=n_{\mathrm{n}} \sigma r^{2}$, 
where $\sigma=5 \times 10^{-20} \mathrm{~m}^{2}$ is the electron-neutral cross-section for 5$\mathrm{eV}$ electrons with water molecules, as used by, for example, Mandt et al. (2016) and Henri et al. (2017). In order to relate observations to how collisional the electrons are at the spacecraft position $r$, it is possible to give this position in units of $L_{\mathrm{c}}$ as

$R^{*}=\frac{r}{L_{\mathrm{c}}}=\frac{1}{n_{\mathrm{n}} \sigma r}$

We can note that Rosetta orbits most of the time outside this boundary, $R^{*}>1$ (Mandt et al. 2016, fig. 5). This does not mean that the electrons are completely collisionless, but a higher value of $R^{*}$ indicates a lower rate of collisions.

Not only is the comet weakly active and the plasma environment not fully developed, but the plasma environment turns out to be very unstable. The most prominent example is the detection of the 'singing comet waves', with frequencies of 10-100 $\mathrm{mHz}$ and very large amplitude, $\mathrm{d} B / B \sim 1$ (Richter et al. 2015; Koenders et al. 2016), in the low-activity stages of the mission, but strong variations in all plasma parameters were found throughout the mission.

The plasma density is highly variable in all regions investigated by Rosetta (Edberg et al. 2015; Odelstad et al. 2015; Stenberg Wieser et al. 2017). It is smoother inside the diamagnetic cavity (Goetz et al. 2016b, 2016a; Henri et al. 2017), but large density fluctuations have been observed there as well (Hajra et al. 2018). Outside the diamagnetic cavity, pulses of higher density are regularly observed. They vary in duration but are typically of the order of a few to a few tens of seconds in the examples given by Eriksson et al. (2017).

The boundary of the diamagnetic cavity seems to be unstable. Hybrid simulations by Koenders et al. (2015) predicted that filaments of plasma are cut off from the diamagnetic cavity and move into the magnetized region outside, with the instability being most prominent in the plane perpendicular to the external magnetic field. These then are sharp density increases, much like the pulses we see, or filaments that move tailwards. Rosetta observations also show that many cavity observations are short, around a minute or even less, and Henri et al. (2017) suggests that these structured can be interpreted as finger-like structures extending out from a central cavity into the surrounding magnetized plasma. It can be noted that the filaments found by Koenders et al. (2015) in the hybrid simulations do not have a zero magnetic field, and so cannot be directly identified with the cavity fingers suggested by Henri et al. (2017). Henri et al. (2017) also show that the occurrence statistics of these brief cavity observations are well organized by $R^{*}$, pointing to the importance of the electron collisionality for the cavity physics.

In this paper, we investigate the pulses of high plasma density observed outside the diamagnetic cavity by Eriksson et al. (2017), as opposed to the plasma density pulses inside the diamagnetic cavity studied by Hajra et al. (2018). These pulses, where the density is higher than the background density, have a relatively short duration, typically a few seconds to a few tens of seconds, as seen from the spacecraft. However, longer durations (up to about $10 \mathrm{~min}$ ) have been observed. As shown by Eriksson et al. (2017) they are very common, and it is therefore important to understand them and their role in the comet plasma environment. Four events are discussed in detail (Section 3), based on data from several Rosetta instruments (Section 2.8). In order to investigate their relationship to the electron cooling, we also perform a statistical investigation of their occurrence (Section 3.4).

\section{METHODS AND INSTRUMENTS}

\subsection{RPC-LAP}

The main instrument used in this study is the RPC (Rosetta Plasma Consortium; Carr et al. 2007) LAP (LAngmuir Probe) instrument (Eriksson et al. 2006). It uses two separate spherical Langmuir probes (LAP1 and LAP2) that are identical and can be operated in different modes. They are mounted on booms $(2.2$ and $1.6 \mathrm{~m}$, respectively) that separate the probes by $5 \mathrm{~m}$.

LAP can be put into different modes for accessing different parameters and for adapting the measurements to the plasma conditions and available telemetry. The main modes are (1) bias voltage sweep, (2) constant bias potential and (3) constant bias current, including the floating probe, which is mostly used for setting the two probes together in an E-field mode (see Eriksson et al. 2006 and Eriksson et al. 2017 for details). The mode used in this study is when the probe is set to a constant bias potential, attracting either electrons or ions (2). As the currents carried by plasma particles to a Langmuir probe are proportional to the plasma density, this mode is useful for high-time-resolution measurements of the plasma density dynamics. We will also use LAP modes with at least one probe measuring voltage when floating; that is, not exchanging any current with the surrounding plasma and providing a measure of the spacecraft potential (Odelstad et al. 2017).

The operational modes are defined and operated by so-called macros. These are short scripts that run the instrument in the appropriate mode. A macro is typically run for a time span from a few hours to about a day, until a new macro is commanded. The interval in which a particular macro runs is called a macro block. The macro blocks are the basic divisions in time we use in this study.

In order to obtain information on the pulses that is as complete as possible, we will present data from several events (see Section 3) with LAP in different operational settings, measuring different quantities. The macros used here are internally identified as 624 and 914 . We will refer to them by the more descriptive designations EI (for electron-ion) and II (for ion-ion), respectively. In macro EI, LAP1 is set to a bias potential of $+30 \mathrm{~V}$ and LAP2 is set to $-30 \mathrm{~V}$, so the two probes sample mainly electrons and ions, respectively. This is the mode used in the example given by Eriksson et al. (2017), where the simultaneous increase in both these currents showed that the pulses observed by LAP result from real increases in plasma density. The probe currents are continuously recorded at $57.8 \mathrm{~Hz}$, except for short data gaps (less than a second) every $32 \mathrm{~s}$ and breaks for probe bias sweeps for more complete plasma characteristics at 160-s intervals. Macro II is similar to EI but has both probes set to $-30 \mathrm{~V}$, which means that the current they measure results from plasma ions attracted to the probe, with some addition from the almost-constant or at least slowly varying photoelectron emission (Johansson et al. 2017) from the probe. Because the two probes operate in the same way, the two signals can be directly compared. For one event, macro 802 was used, hereafter called VV (for voltage-voltage). Here both LAP probes are used in voltage mode with no bias current or voltage applied, and the potential of the freely floating probes is measured at $57.8 \mathrm{~Hz}$. As shown by Odelstad et al. (2017), this gives a measure of the (negative) spacecraft potential, $V_{\mathrm{s}}$, which in turn is sensitive to the plasma density.

Neither of the LAP modes above gives information on the absolute value of the plasma density unless they are complemented by some assumption or measurement of the electron temperature. Such temperatures can be derived from LAP in sweep mode (Eriksson et al. 2017). Another way is to use the proportionality of the probe 
current to plasma density together with the independent plasma density measurement provided by the RPC Mutual Impedance Probe (MIP) instrument for calibration. This will be done in Section 2.7.

\subsection{RPC-MIP}

The MIP (Trotignon et al. 2007) is also part of the RPC. It transmits a signal at various frequency steps and observes the response at the same frequency. The plasma density is then retrieved by on-ground identification of characteristics, such as the resonance peak, of the mutual impedance spectrum (Gilet et al. 2017). MIP is considered to provide a reliable measurement for the total plasma density when a resonance peak can be clearly identified in the mutual impedance spectra. This means in practice that the plasma density has to be above or around $100 \mathrm{~cm}^{-3}$ (the limit being dependent on the electron temperature). MIP data (usually available at a time resolution of between 4 and $32 \mathrm{~s}$ ) can be used to calibrate the LAP current sampled at a higher rate $(57.8 \mathrm{~Hz}$ for the data presented here) to a plasma density value using a linear fit. This also extends the density range to values too low for the MIP to measure. LAP observations can give the electron density even if the MIP can not. This is discussed further in Section 2.7.

\subsection{RPC-ICA}

The ICA (Nilsson et al. 2007) is the Ion Composition Analyzer of the RPC. It measures the mass-separated energy distribution function of positive ions from a few electronvolts up to $40 \mathrm{keV}$ within $45^{\circ}$ of the detector plane, so the solid angle coverage is about $2 \pi \mathrm{sr}$. ICA is mounted on the spacecraft so that both the Sun and the comet nucleus are in the field of view of the instrument during nominal pointing when all imaging instruments on Rosetta look towards the nucleus (Nilsson et al. 2015). It can usually be assumed to cover the most important flow directions from which the ions are expected to come, namely from the nucleus or streaming with an anti-sunward component. The angular distribution of cometary ion flow throughout the mission and detailed examples are given in Nilsson et al. (2017). If there is a population of low-energy ions and if the spacecraft potential, $V_{\mathrm{s}}$, is sufficiently negative, the ICA ion observations give the spacecraft potential. For a negative $V_{\mathrm{s}}$, all ions will have been accelerated to an energy of at least $e V_{\mathrm{s}}$ when they reach the ICA, so the lowest energies detected give $V_{\mathrm{s}}$ (Odelstad et al. 2017). In the later part of the mission, the ICA often used a mode with limited energy range but high time resolution (Stenberg Wieser et al. 2017). As this mode has sufficient time resolution for the pulses in which we are interested, this is the mode used in this study.

\subsection{ROSINA-COPS}

From ROSINA (Rosetta Orbiter Spectrometer for Ion and Neutral Analysis; Balsiger et al. 2007) we use data from the nude gauge of the COPS (COmet Pressure Sensor). This instrument measures the total neutral gas density. The primary measured quantity is the ion current to a small sensor. However, the ions are created by ionizing the neutral gas in a volume designed to let only the gas in and to keep the plasma out by electrostatic means. The resulting current is thus proportional to the number density of the neutral gas. Nevertheless, sufficiently energetic plasma particles may enter COPS at times (Tzou 2017), and so we will not consider COPS signatures of fast variation as real neutral gas density variations. Our main use of COPS is instead to provide the large-scale neutral gas background, which is important not only as the source of the comet ionosphere but also for cooling the electron gas.

\subsection{RPC-MAG}

MAG, the RPC fluxgate magnetometer (Glassmeier et al. 2007; Richter et al. 2015), measures the magnetic field vector. The MAG data used here are sampled at $20 \mathrm{~Hz}$. No noise reduction has been applied to the data, only calibration by removing the mean of the magnetic field in each component inside the diamagnetic cavity, when available. Our chief interest in MAG data is for comparing the magnetic signal with the local density fluctuations, but we also use MAG to see if the phenomena we study are organized by the magnetic field. Owing to sensor temperature sensitivity and noise from spacecraft systems, some quasi-constant offsets may sometimes remain in MAG data. This is not a problem for identifying transient structures, but some caution is needed when calculating angles of the magnetic field when the magnetic field is weak.

\subsection{Event selection}

The main focus of this study is to provide details of the strong plasma density fluctuations seen as high-density pulses by Rosetta. These were most common in the months around perihelion (Eriksson et al. 2017), so we concentrate on events during the highest cometary activity phase of the Rosetta mission. The events are chosen from 2015 autumn, within a few months after perihelion (2015 August 13). Data from the months leading up to perihelion could also have been used. However, new LAP operational modes tailored to the conditions found around perihelion were uploaded in early August and MIP operations were also optimized in that time-frame, so the post-perihelion phase offers better prospects.

We concentrated on periods when all the used RPC instruments operated in their high-telemetry rate, known as burst mode, in order to obtain high-time-resolution data. In order to avoid the effects of changing probe illumination, stable spacecraft pointing was required for all events, with only minuscule changes (a few degrees) in attitude. With these constraints, at least one example interval was identified for each of the LAP modes EI, II and VV, as each of these can illuminate different aspects of the pulses.

Rosetta spent most of its time in the terminator plane (Taylor et al. 2017), so selecting events randomly may give the impression that the pulses only exist there. To show that this is not the case, we made sure that some of the included events were from different solar zenith angles (SZA, also known as the phase angle).

The nucleus rotation period is close to $12 \mathrm{~h}$, and the outgassing varies over the comet surface and with illumination. Intervals of $12 \mathrm{~h}$ are therefore desirable, but some shorter intervals had to be accepted. Finally, periods when ICA was operated in its low-energy high-time-resolution mode were preferred, although not all events could be chosen to include such data. After weighting all the requirements, we obtained the set of four events listed in Sections 3.1-3.3 to illustrate various features of the pulses.

Table 1 gives an overview of the events investigated. The neutral density $n_{\mathrm{n}}$ from COPS and the distance $r$ of Rosetta to the centre of the nucleus change during the intervals, but average values are given. From these values we also calculate an approximate production rate (molecules per second) relevant for the event, by assuming spherica symmetry and a gas outflow speed $u=1 \mathrm{~km} \mathrm{~s}^{-1}$, as $Q=4 \pi r^{2} n_{\mathrm{n}} u$.

Fig. 1(a) shows the 3D position of Rosetta at the times of the events we investigated. We use the CSEQ (cometocentric solar equatorial) coordinate system. The comet nucleus is marked by the 
Table 1. An overview of the events presented in this paper. Given are the date, time, LAP macro, as well as approximate values for the COPS neutral density $n_{\mathrm{n}}$, radial distance $r$, gas production rate $Q$ and solar zenith angle SZA.

\begin{tabular}{lccccccc}
\hline Date & $\begin{array}{c}\text { Block time (start) } \\
{[\mathrm{h}]}\end{array}$ & Macro & $\begin{array}{c}n_{\mathrm{n}} \\
{\left[10^{7} \mathrm{~cm}^{-3}\right]}\end{array}$ & $\begin{array}{c}r \\
{[\mathrm{~km}]}\end{array}$ & $\begin{array}{c}\mathrm{Q} \\
{\left[10^{27} \mathrm{~s}^{-1}\right]}\end{array}$ & $\begin{array}{c}\text { SZA } \\
{\left[{ }^{\circ}\right]}\end{array}$ & Remarks \\
\hline 2015 Oct 24 & $06-16(14)$ & EI (624) & 1 & 400 & 20 & 60 & Both probes sunlit \\
2015 Nov 15 & $00-12(08)$ & II (914) & 2 & 140 & 5 & 60 & - \\
2015 Nov 15 & $12-00(12)$ & VV (802) & 2.5 & 160 & 8 & 60 & E-field mode \\
2015 Nov 20 & $20-00(22)$ & EI (624) & 3 & 150 & 8 & 90 & - \\
\hline
\end{tabular}

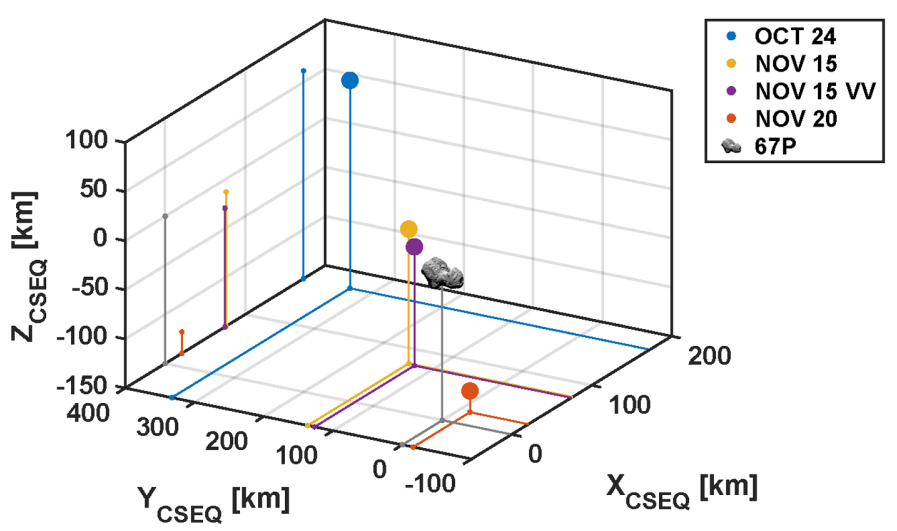

(a) a

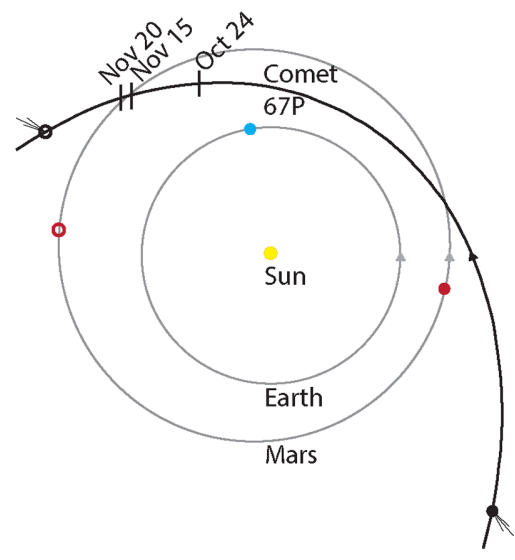

(b) $\mathrm{b}$

Figure 1. (a) Position [km] of Rosetta relative to 67P for the events in Table 1, averaged over the macro blocks, given in the CSEQ (cometocentric solar equatorial) coordinate system. Here the $x$-axis points from the comet to the Sun, the $z$-axis is the component of the Sun's rotation vector, perpendicular to the $x$-axis, and the $y$-axis completes the right-handed reference frame (e.g. described by Edberg et al. 2016). (b) The positions of Rosetta, Earth and Mars during 2015 seen from $90^{\circ}$ above the ecliptic plane, with the position of the selected events marked. The filled circle is the starting position and the empty circle is the final position.

grey circle at the origin. It can be seen that all observations are taken on the day side, namely at positions with a positive $X$-coordinate. This reflects the operational constraint that the spacecraft should not enter the night side of the nucleus. Two of the events are from the terminator plane $\left(X_{\mathrm{CSEQ}} \approx 0\right)$ and the remaining three are from the day side at significant phase angle (solar zenith angle).

Eriksson et al. (2017) provided an overview of the pulse occurrence in LAP data over the full year 2015, spanning 1.25 to 2.5 au. This period can be seen in Fig. 1(b), where solid circles mark the positions of the various objects at the start of 2015, and the empty circle marks the end. The picture also shows the approximate position of $67 \mathrm{P}$ during the chosen events.

\subsection{LAP-MIP cross-calibration}

In contrast to MIP, the current (or voltage) detected by LAP depends primarily on the particle fluxes reaching the probe and (for the spacecraft potential) the spacecraft. For a constant shape of the energy distribution, the fluxes and thus the LAP current should depend only on the density (Eriksson et al. 2017); variations in, for example, temperature will affect LAP. As long as the spacecraft potential is stable, the LAP current will be proportional to the plasma density. The random electron current flowing to a spherical probe at the same potential as the surrounding plasma is proportional to the electron density $n_{e}$, as given by

$$
I_{e, o}=e A_{\mathrm{LP}} n_{e} \sqrt{e T_{e} / 2 \pi m_{e}},
$$

where $e, T_{e}$ and $m_{e}$ are the electron charge, temperature and mass, respectively, and $A_{\mathrm{LP}}$ is the surface area of the probe. A real probe will be at some potential to the plasma, and standard theoretical expressions give a correction factor for this:

$I_{e}=I_{e, o}\left(1+\frac{V_{\mathrm{b}}+V_{\mathrm{s}}}{T_{e}}\right)$

where $V_{\mathrm{b}}$ and $V_{\mathrm{s}}$ are the bias and spacecraft potential, respectively (Eriksson et al. 2017). The ion current to a negative probe obeys a similar expression.

In order to use these expressions to derive the density from the LAP current we have to assume values for parameters such as the spacecraft potential, electron temperature and ion velocity, which are not known at high time resolution and always with some uncertainty. Even though the spacecraft potential $V_{\mathrm{s}}$ is also dependent on the plasma density, we can at least for a limited density range assume that the electron current is proportional to the density, as in equation (3). We then apply a fit

$n_{\mathrm{MIP}}=A\left(I_{\mathrm{LAP}}+B\right)$.

Because in a plasma $n_{e} \sim n_{i}$, we can use the same formula to find the appropriate fit for the ion and the electron current. In order to derive the density from the potential measurement, VV mode, we use the fact that the spacecraft potential is related to the plasma density as (Odelstad et al. 2017; Heritier et al. 2017)

$n \propto \exp \left(-\alpha V_{\mathrm{s}} / T_{e}\right)$ 
Table 2. An overview of the fit parameters resulting from calibrating the LAP currents to the MIP plasma density, according to equations (5) and (7).

\begin{tabular}{|c|c|c|c|c|c|}
\hline \multirow[t]{2}{*}{ Date } & \multirow[t]{2}{*}{ Macro } & \multicolumn{2}{|c|}{ Fit LAP1 } & \multicolumn{2}{|c|}{ Fit LAP2 } \\
\hline & & $A\left[\mathrm{~cm}^{-3} / \mathrm{nA}\right]$ & $B[\mathrm{nA}]$ & $A\left[\mathrm{~cm}^{-3} / \mathrm{nA}\right]$ & $B[\mathrm{nA}]$ \\
\hline 2015 Oct 24 & EI (624) & 0.97 & 0.6 & 60 & -18.3 \\
\hline 2015 Nov 15 & II (914) & 20 & -10.0 & 20 & -10.0 \\
\hline \multirow[t]{3}{*}{2015 Nov 20} & EI (624) & 0.007 & 649.3 & 18.5 & 6.4 \\
\hline & \multicolumn{3}{|c|}{ Fit LAP1 and LAP2 } & & \\
\hline & & $C[\mathrm{eV}]$ & $D\left[\mathrm{~cm}^{-3}\right]$ & & \\
\hline 2015 Nov 15 & VV (802) & 3.3 & 200 & & \\
\hline
\end{tabular}

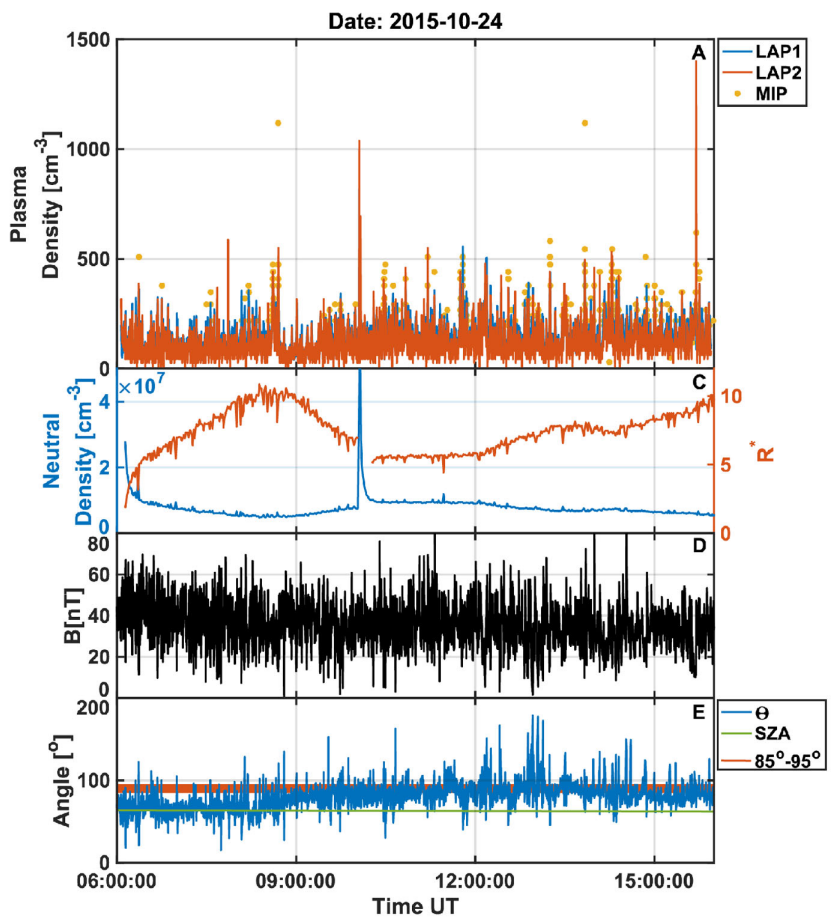

(a) 10 hours

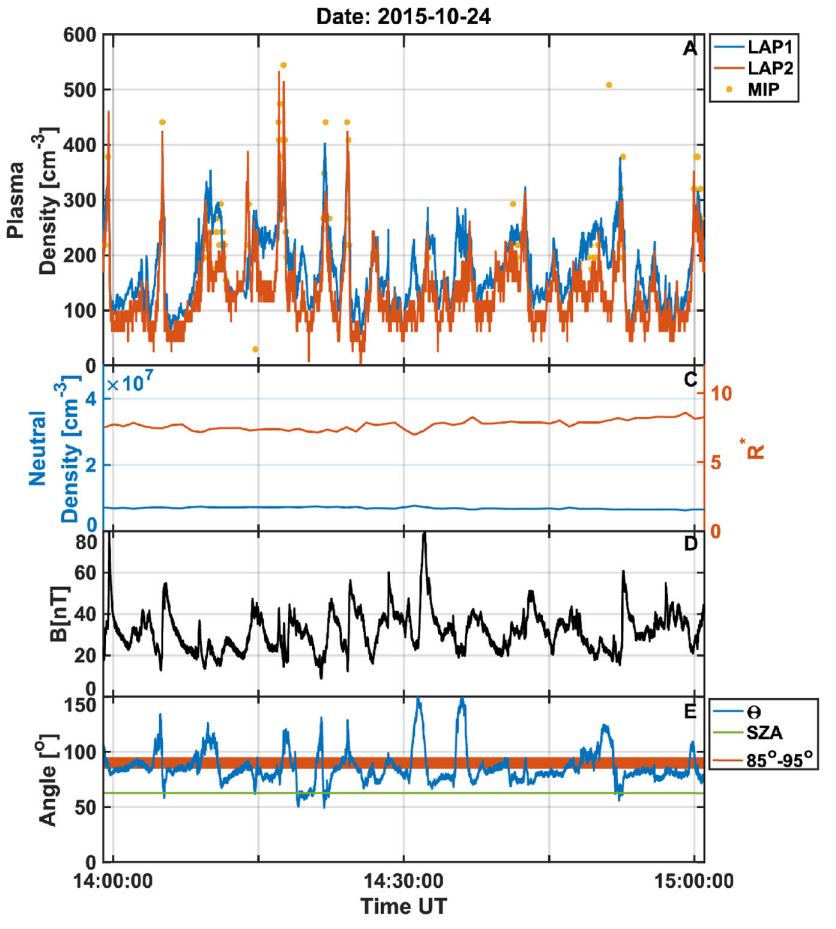

(b) 1 hour

Figure 2. (a) Data for October 24. LAP was in EI mode on this day. (b) One hour from the October 24 data set (14:00-15:00). The panels in both figures are as described in Section 2.8. The data spike in LAP and COPS (Panels A and C) around 10:00 is caused by a spacecraft thruster firing and should be neglected.

so we can perform a linear fit of $\log n$ to the measured probe potential $U_{\text {LAP: }}$

$\log n_{\mathrm{MIP}}=\log D+U_{\mathrm{LAP}} / C$.

Table 2 shows the fits used for calibrating the LAP current and voltage to the density is measured by MIP. As expected, the fit coefficients are different for probes in positive and negative bias potential (E and I modes). We also see a significant difference between events, particularly in the E mode. This is because of different plasma conditions, which in turn cause different spacecraft potentials. This can be seen in equation (4) to change the linear relationship of the density and current.

\subsection{Data presentation}

In order to provide an overview of the coma environment for the selected events (see Section 3, Figs 2 to 6), we present data from
LAP, MIP, ICA (where available), COPS and MAG. The panels are further explained in the following description.

Panel A: This panel shows the plasma density by both LAP probes (blue and red) and MIP (orange). The calibration is explained in Section 2.7.

Panel B: The ICA high-resolution data are plotted, where available. The vertical axis shows the energy in electronvolts, and the colour scale gives the flux of ions for each energy channel.

Panel C: On the left vertical axis of the plot (blue curve) we show the neutral density as derived from ROSINA COPS. The right vertical axis (red curve) shows the spacecraft position relative to the electron exobase distance, $R^{*}$ (equations 1 and 2).

Panel D: This panel shows the magnetic field measured by MAG in CSEQ coordinates. $B_{\text {tot }}$ is shown in black, $B_{x}$ in red, $B_{y}$ in green, and $B_{z}$ in blue.

Panel E: This panel shows the angle (blue) between the CometRosetta vector and the local magnetic field, for $B_{\text {tot }}>10 \mathrm{nT}$, as 


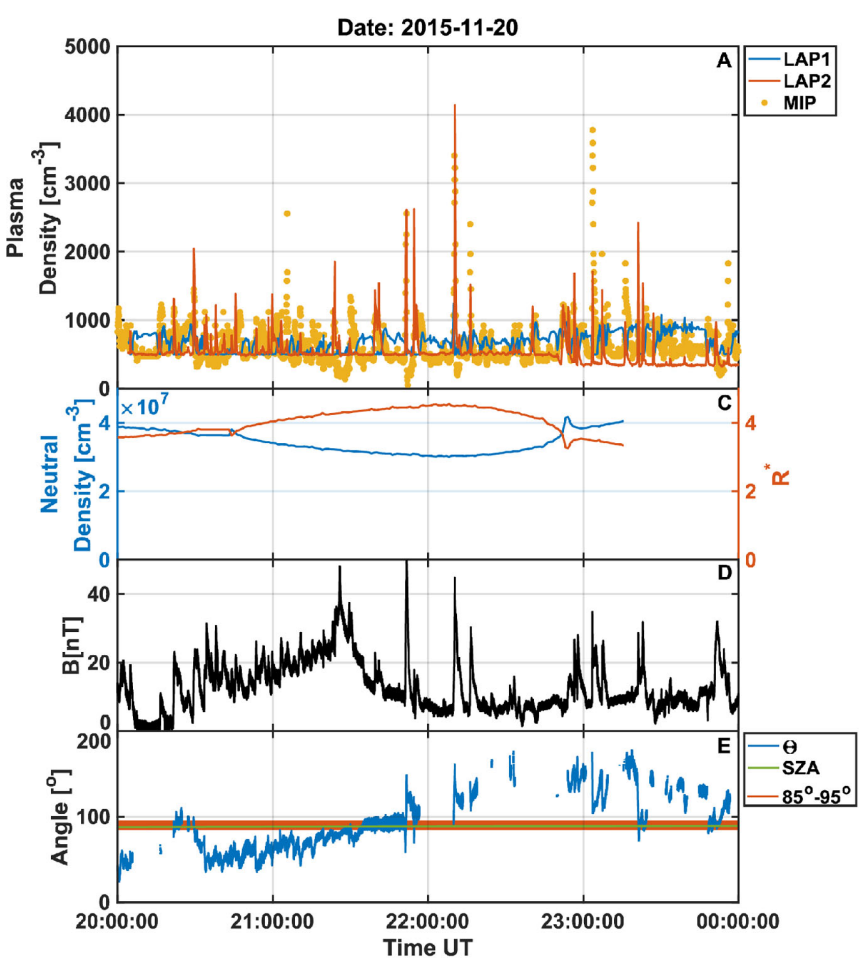

(a) 4 hours

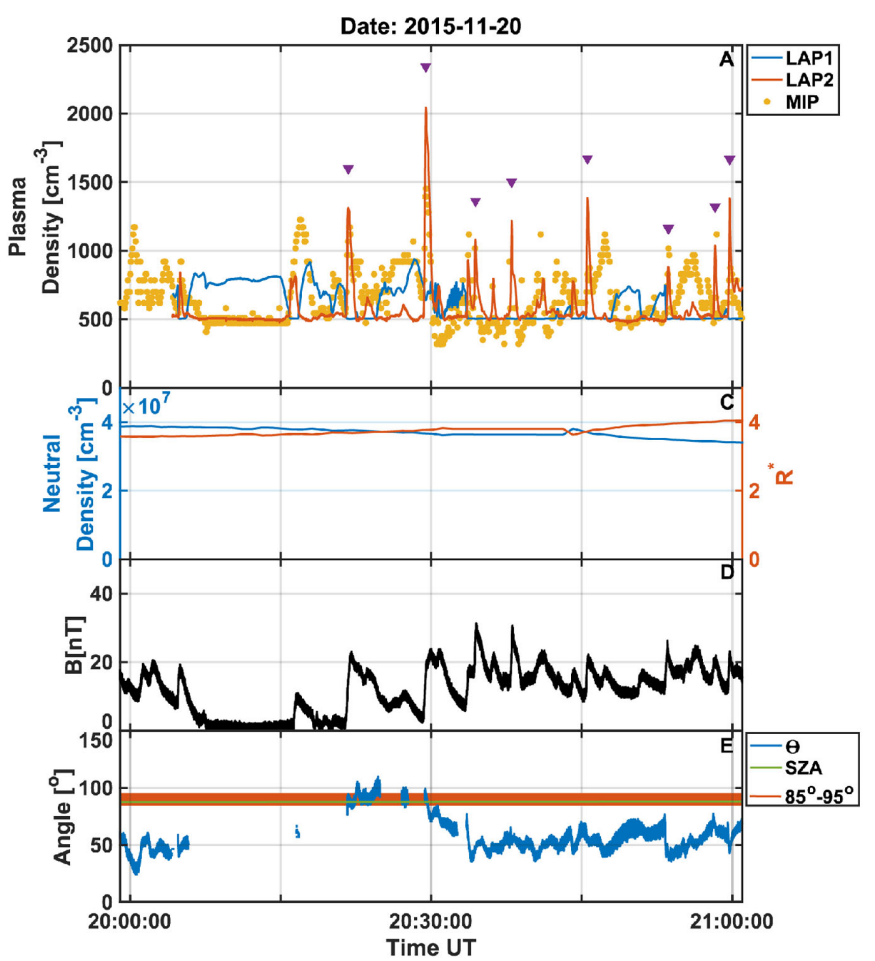

(b) 1 hour

Figure 3. (a) Overview data for November 20. LAP is in EI mode for this short macro block. (b) One hour of data from November 20, 22:00-23:00. The purple triangles show the pulses that were detected with the automated algorithm used in Section 3.4. The panels for both figures are as described in Section 2.8.

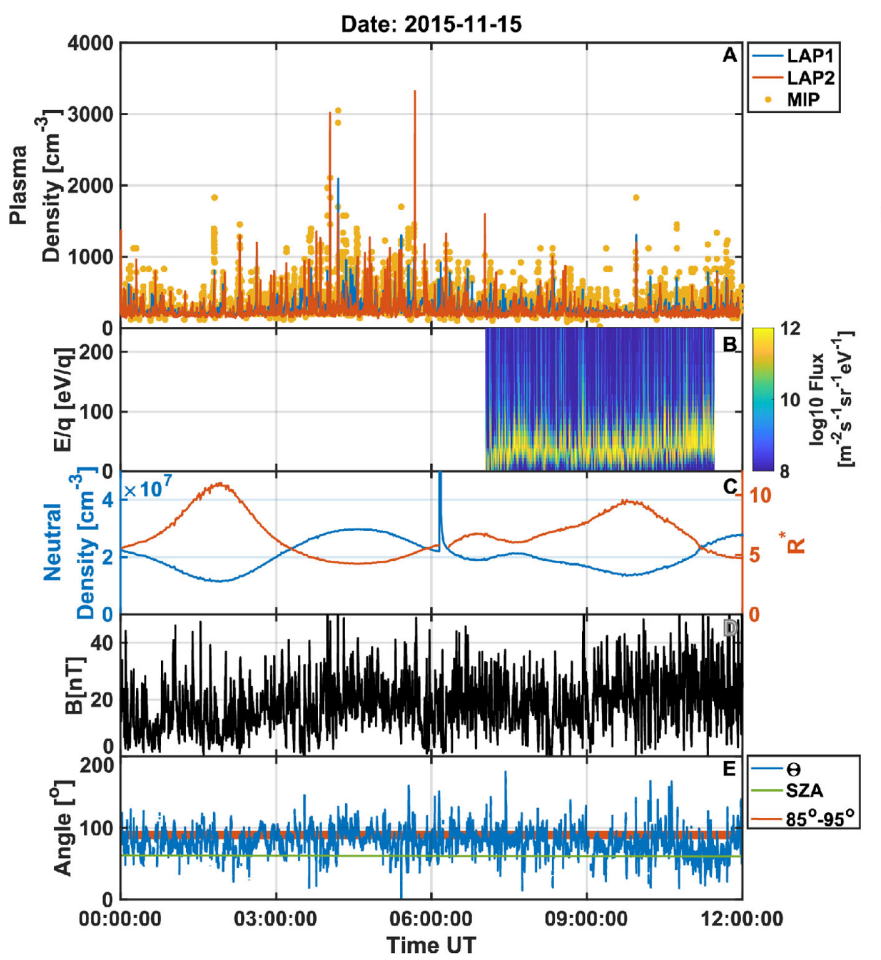

(a) 12 hour

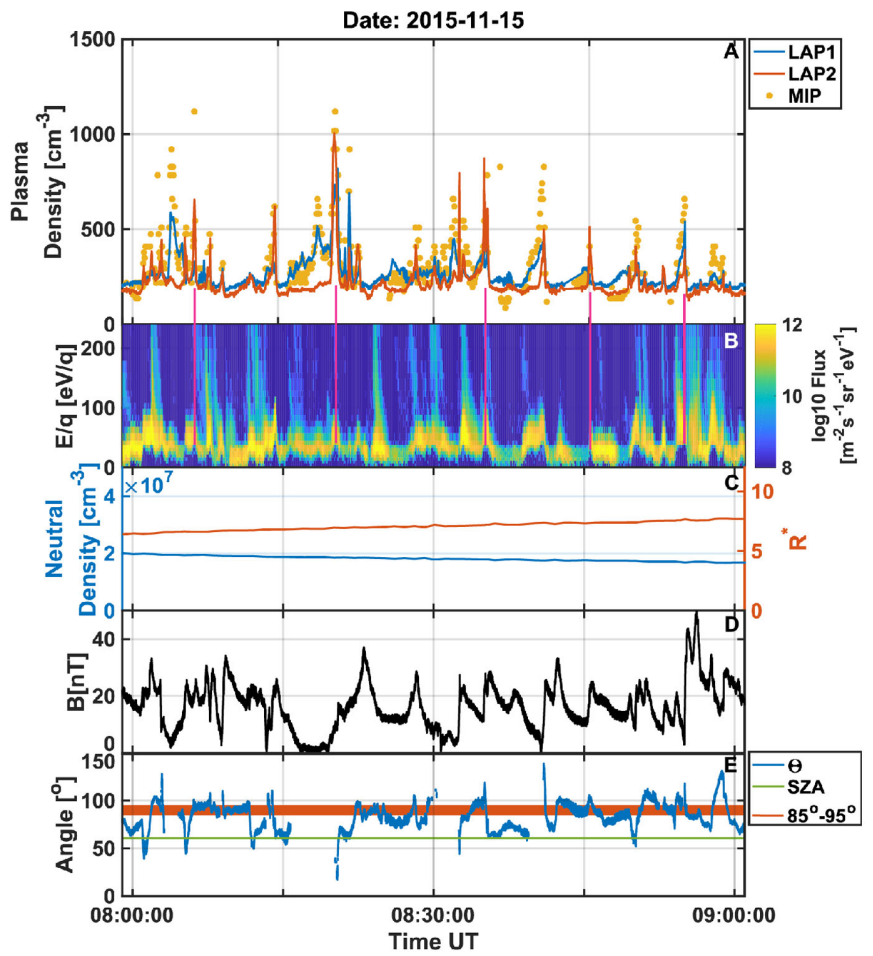

(b) 1 hour

Figure 4. (a) Overview data for November 15 in the morning. Here LAP is in II mode. (b) One hour (08:00-09:00) of data from November 15. The panels for both figures are as described in Section 2.8. The pink lines show the correspondence between ICA's lower-energy cutoff and the pulses. 


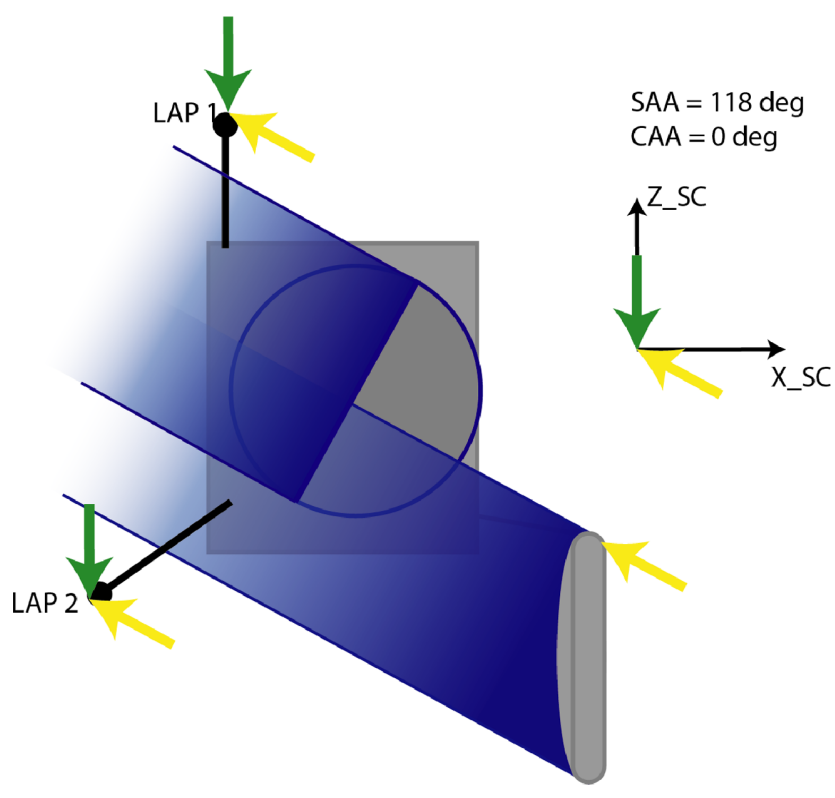

Figure 5. Rosetta pointing on 2015 November 15 . The grey box is the spacecraft body and the grey object to the lower right is the high-gain antenna. The solar panels extend into and out of the page (along the $Y$-axis), as does LAP1. The solar (SAA) and comet (CAA) aspect angles are the angles of the Sun and the nucleus with respect to the $+Z_{s}$-axis. Yellow and green arrows denote the flow directions from the Sun and the comet nucleus, respectively. The shadows of the solar panels and the high-gain antenna are indicated. For further explanations, see text.

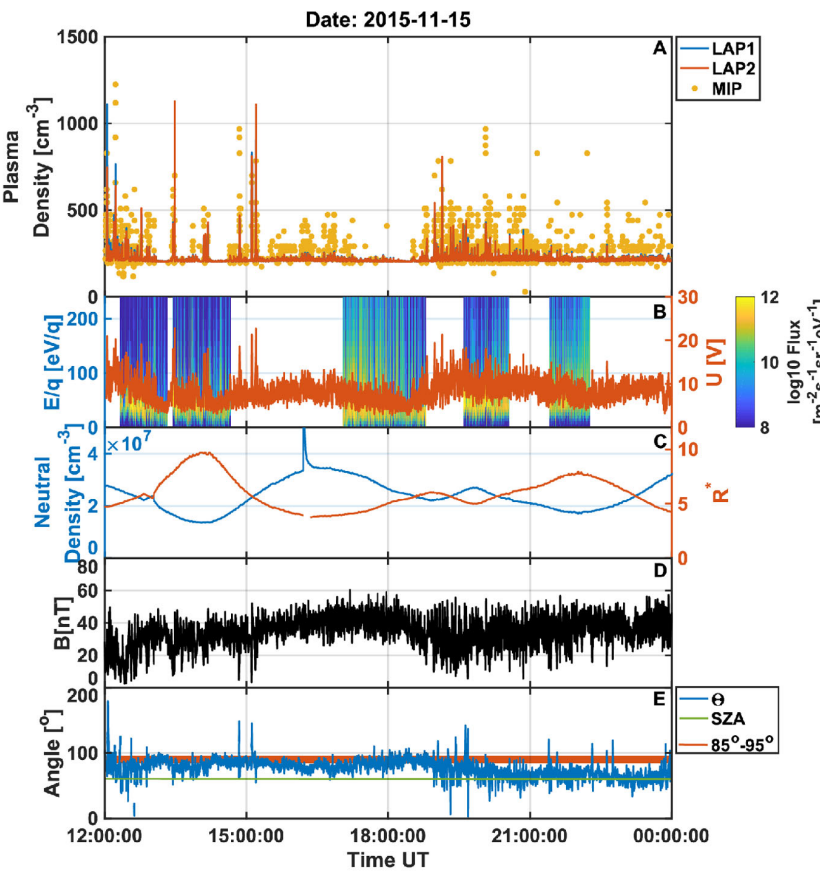

(a) 12 hour well as the solar zenith angle (green). The red horizontal bar marks angles between $85^{\circ}$ and $95^{\circ}$.

\section{OBSERVATIONS}

\subsection{October 24}

The first event we present here is 2015 October 24. As seen in Fig. 1(a), Rosetta was in the positive- $Z$ hemisphere of the comet, on the evening side of the nucleus at a solar zenith angle of about $60^{\circ}$ and at a distance of $400 \mathrm{~km}$. Evening (and morning) is here defined in terms of local time at the point on the rotating nucleus directly below Rosetta. Fig. 2(a) shows an overview of the full macro block with LAP in the EI mode. LAP1 samples electrons and LAP2 samples ions. Both currents have been fitted to the MIP plasma density as described in Section 2.7. The spike seen in LAP (Panel A) and COPS (Panel C) data at around 10:00 was caused by a spacecraft thruster firing and should be neglected. We will not go into further detail about the effects of these manoeuvres on various instruments. We note only that these effects are common and usually decay within a few tens of minutes (Tzou 2017). The plasma density in LAP1 and 2 seem to follow each other well, which is more apparent in the zoom in Fig. 2(b). This figure shows a 1-h interval of Fig. 2(a), and many density spikes show up clearly in both probes. The plasma density, $n$, is a few hundred $\mathrm{cm}^{-3}$ inside the pulses, while it is lower than $100 \mathrm{~cm}^{-3}$ outside. MIP is not able to resolve the lower density outside the pulses, but together MIP and LAP cover the density range of interest.

Some simultaneous variations may also be present in the neutral gas (COPS, Panel B), but they are much smaller ( $<10$ per cent) than the plasma signatures (factor of 5 in LAP current). Because it is known (Tzou 2017) that COPS may sometimes react to plasma

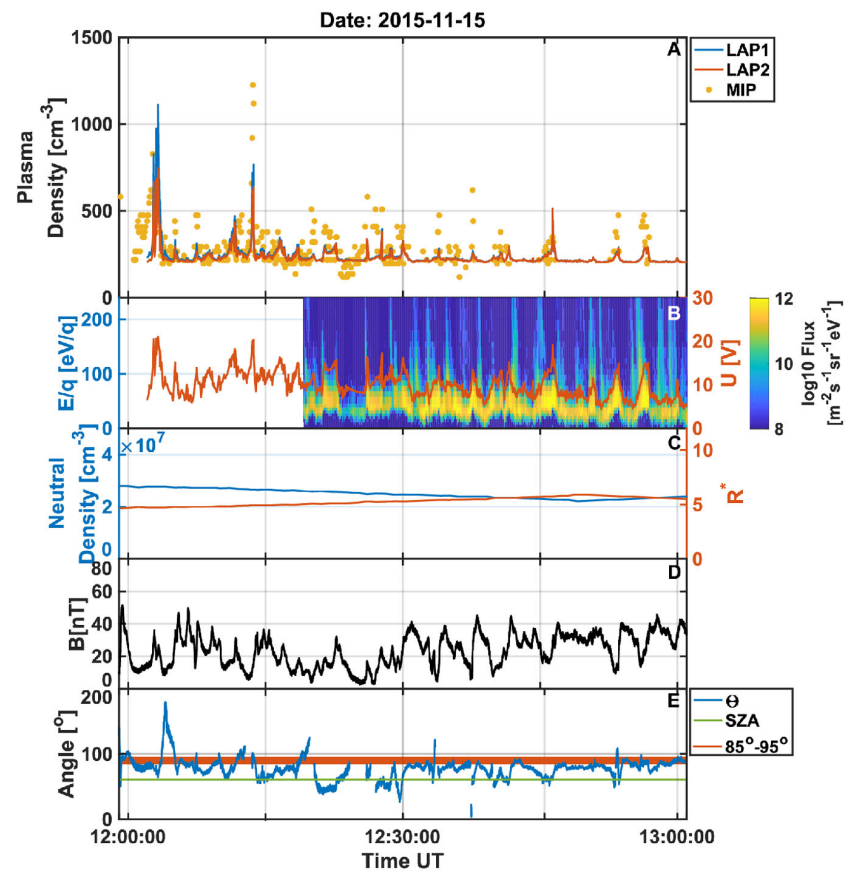

(b) 1 hour

Figure 6. (a) Second part of data from November 15. MIP, LAP are in VV mode. ICA, COPS and MAG show plasma dynamics similar to those in the previous part of the day. Thus plasma dynamics are comparable to 4(a). Because LAP is in E-field mode producing voltages, it is shown in Panel B together with the ICA measurements with the energy scale in electronvolts, with some stretching of the voltage range for clarity. There are data gaps in ICA until 12:18, 14:50-17:00, 18:50-19:40, 20:15-21:20 and 22:20-23:10. (b) One hour on November 15 (12:00-13:00). The panels for both figures are as described in Section 2.8. 
variations, we do not interpret these small variations as being caused by real fluctuations in the neutral gas. The COPS data indicate that the spacecraft is at about 8 times the cometocentric distance of the electron exobase (red line in Panel C). Because diamagnetic cavity observations were mostly found closer to the electron collsionopause (Henri et al. 2017), this also indicates that Rosetta is quite far outside the diamagnetic cavity. In fact, no diamagnetic cavity boundary crossing was identified by Goetz et al. (2016a) during the whole month of October 2015. This is because a dayside excursion occurred during this month, and Rosetta was far away from the nucleus.

The magnetic field (Panel D) generally varies in the same way as the LAP and MIP data, with density and magnetic field strength increasing together, further verifying the plasma nature of the structures. However, the pulses are more asymmetric in the magnetic field than in density, with a sharp leading edge and a slow decay. This asymmetry is also seen in the ion energy and spacecraft potential (Stenberg Wieser et al. 2017). As shown by Hajra et al. (2018) for observations of similar structures just outside the diamagnetic cavity, this asymmetry can often also be found in the density, but it is not obvious here.

\subsection{November 20}

Fig. 3(a) shows the data from November 20. Rosetta was in this case close to the terminator on the morning side of the nucleus at about $150 \mathrm{~km}$ distance (Fig. 1a). As can be seen in Panel C, the neutral density is much higher in this event. This is not only because Rosetta is closer to the nucleus, but also because it is in the southern hemisphere, which during this time of the mission was in summer and thus more active (Hansen et al. 2016). Hence Rosetta is only at about 4 times the distance of the electron exobase. The plasma density is also much higher, resulting in good MIP density estimates for all of the day. As expected (Henri et al. 2017), when close to the exobase Rosetta is sometimes inside the diamagnetic cavity, and 82 cavity observations are listed for this day by Goetz et al. (2016a).

As in the previous event (see Section 3.1), LAP is in EI mode with LAP1 at a positive bias voltage to sample electrons and LAP2 at a negative one to sample ions. Interestingly, the densities measured by LAP1 and LAP2 seem to be anticorrelated. This is because the plasma density is higher on this day than on October 24, which is mainly because Rosetta is closer to the nucleus and in the southern hemisphere. At higher plasma densities, the spacecraft potential is usually more negative, about $-12 \mathrm{~V}$ (Odelstad et al. 2015). If the spacecraft potential is more negative than the positive bias voltage applied to the probe, then the probe is not positive with respect to the plasma and electrons cannot be collected. Therefore when the plasma density increases, the electron current to the probe can decrease, and therefore the density derived from LAP1 decreases. The effect is stronger if the electrons inside the pulse are colder than those outside (Eriksson et al. 2017), as they then will have even less kinetic energy to overcome the more negative $V_{\mathrm{s}}$. An example of direct measurement of a more negative spacecraft potential in pulses will be given in Section 3.3.

For a negatively biased probe, such as LAP2 in this case, a more negative spacecraft potential inside the pulses serves to increase the ion current. This adds to the direct increase of the ion current owing to the higher density. The ion current measurements, at negative bias potential, therefore always correlate with the plasma density. We can see this in Panel A, where MIP and LAP2 density variations agree very well. Negatively biased probes are therefore safer to use for obtaining plasma density in a dense plasma. However, when the ion current is much lower than the electron current, that is, when the plasma density is low, the signal-to-noise ratio in the ion current is low. Fortunately, the spacecraft potential depends on density and will be low in such cases, so the density variations can be observed in the electron current to a LAP probe at positive bias (as in the October 24 event, Section 3.1).

Considering a 1-h interval, shown in Fig. 3(b), it can be seen that there are coincident pulses in the magnetic field and plasma density. The pulses thus appear to be compressional in nature, as discussed by Hajra et al. (2018). During this hour, Rosetta was inside the diamagnetic cavity between 20:08 and 20:16 (Goetz et al. 2016b). The angle between the $B$-field and nucleus direction is within about $45^{\circ}$ of the perpendicular, and there is little change of this angle in the pulses.

\subsection{November 15}

\subsubsection{Morning}

November 15 is particularly interesting because the first half of the day has readings in II mode and the second half in VV mode, both with (some) coverage of ICA high-resolution data. COPS neutral gas density data are basically identical for the two 12-h intervals, and therefore the magnetic field seen by MAG and the plasma density seen by MIP are also quite similar, so the two macro blocks are comparable to each other. The VV data can be used to investigate how the spacecraft potential, $V_{\mathrm{s}}$, varies inside a pulse.

During this day, Rosetta was close to the equatorial plane in CSEQ coordinates. The solar zenith angle is about $60^{\circ}$ and the cometocentric distance is about $150 \mathrm{~km}$ (Fig. 1a). Fig. 4(a) and (b) show the full 12-h interval and a zoom-in to one hour where LAP operated macro II, measuring the ion density on both probes. Fig. 4(a) shows the familiar variations and pulses in the LAP probe density. The plasma density reported by MIP is much higher than that for October 24 and about half the value of the November 20 event. Rosetta is about 5 times as far from the nucleus as the electron exobase, and there are only two diamagnetic cavity sightings on this day, each of about $2 \mathrm{~min}$ at around 05:50 and 08:18 (Goetz et al. 2016a).

Fig. 4(b) indicates that, despite operating identically, the LAP probes collect slightly different densities. It seems unlikely that this could be explained by very small scales of plasma structure, as electrons inside the pulses last for several minutes and are expected to travel at least as fast as the neutral gas, or at about $1 \mathrm{~km} \mathrm{~s}^{-1}$ (Vigren et al. 2017). More probably the difference is caused by the spacecraft attitude, with LAP2 being sometimes in the wake of the spacecraft, depending on the plasma flow direction, as discussed below.

Fig. 5 shows the pointing of Rosetta with respect to the comet nucleus and the Sun during this day. The yellow and green arrows illustrate the flow directions of solar photons and cometary ions, respectively, assuming that the ions flow radially from the nucleus. Both of these directions varied by less than $2^{\circ}$ during the day. The solar panels are always kept perpendicular to the solar direction, meaning that their edges move along the blue circle. LAP1 is mounted on a boom protruding diagonally out of the paper (Eriksson et al. 2006), and can be in shadow only if it is behind the solar panels, which it never is in this event. The line from the Sun to LAP2 could, on the other hand, be blocked either by the spacecraft body or by the high-gain antenna (seen in the lower right), but we can see that this does not happen here. Therefore both probes are sunlit during all of this day. 
There could also be a wake effect because of a radial ion flow from the comet (green arrows). It can be seen that there is no obstacle directly blocking the flow to any of the probes in this case. However, while LAP1 is on a boom protruding towards the nucleus and should experience an unperturbed plasma flow as long as the radial component is directed away from the nucleus, LAP2 could experience some kind of bow wave or similar extending from the forward edge of the spacecraft. From Fig. 5, it can be seen that a turn of the flow (green arrow) by about $20^{\circ}$ in the clockwise direction would be sufficient to bring LAP2 into a sharply defined wake with edges parallel to the flow direction. In reality, a region roughly defined by a Mach cone from the spacecraft edges will be perturbed (Hastings 1995), so LAP2 may very well see such disturbances. That the plasma flow may depart from the exactly radial direction from the nucleus further increases the possibility of wake effects on LAP2. A few nucleus radii away from the nucleus, kinetic and hydrodynamic models agree that the neutral gas flow must be close to radial from the nucleus (Tenishev et al. 2008; Bieler et al. 2015), but the coupling of gas and plasma is expected to be far from perfect (Vigren et al. 2017), particularly at small scales. That this coupling is not perfect at least at small scales is obvious when comparing the large plasma density variations seen in Panels A with the smoother neutral density in Panels C. Wake effects and changing flow directions are possible sources for the differences between the two LAP probes in Panel A of Fig. 4(b), particularly as LAP2 usually observes the lower density.

ICA high-resolution data are available for this event (Fig. 4b). The density pulses detected by LAP can be seen to correspond to ICA increases in ion flux and ion energy (B). The ion energy increase in a pulse can be at least partly explained by the more negative spacecraft potential, which accelerates ions towards the spacecraft. The ion flux should increase with the density. When looking in more detail, it appears that there is a good correspondence between increases in ICA's lower-energy cutoff (lower edge of yellow region; see pink lines for examples) and LAP density pulses, as is expected for ions accelerated by the spacecraft potential that become more negative with increasing density (a few examples are indicated by vertical lines). However, the ICA ion flux can intensify or spread to higher energy with little or no corresponding density increase (LAP density pulse). Examples of this can be seen at around 08:24 and 08:47. Such signatures can be interpreted as ions accelerated at some distance from the spacecraft and now reaching it with no or a moderate density increase. They share the characteristics of type 5 in the classification of short-lived ICA ion features by Stenberg Wieser et al. (2017).

As in the previous events, the magnetic field shows strong signatures coincident with the plasma density and ion flux enhancements. Also as in previous events, the magnetic field direction between pulses is at quite a large angle to the direction to the nucleus, but the pattern at the pulses is more aligned with the nucleus direction. This is similar to the type 5 signatures in ICA (Stenberg Wieser et al. 2017)

\subsubsection{Afternoon}

During the second half of this day, LAP operated both probes in floating mode (VV), as shown in Fig. 6(a) and (b). The quantity measured here is the probe potential with respect to the spacecraft body, which gives an estimate of the negative of the spacecraft potential (Odelstad et al. 2017). Comparing the data from MIP (Panel A), ICA (B), COPS (C) and MAG (D) with what we found in the first half of the day (Fig. 4a and b), we find similar signatures. In particular, Panel B shows that the pulses in the ICA spectra are seen in LAP both in probe currents (in the morning) and in the probe voltage (afternoon). The LAP1 and LAP2 voltages calibrated to MIP plasma density data, as described in Section 2.7, are shown together with the MIP data in Panel A. It can clearly be seen that the spacecraft becomes more negative in the pulses of higher density, as suggested in Sections 3.2 and 3.3.1. The two LAP probes observe very similar potentials when in this mode, while the probe currents measured in the II mode were seen to differ more between the probes. This can be understood as the voltage picked up by the probes in VV mode being dominated by the potential of the spacecraft as a whole, which is a common property of both probes, while the currents in II mode depend more strongly on the local plasma conditions at each probe, which as noted in Section 3.2 can be influenced by for example wake effects. Nevertheless, there is some difference between the two probe voltages. This difference was used by Karlsson et al. (2017), who derived the electric wavefield in this particular event and found waves in the lower-hybrid frequency range on the edges of the pulses.

\subsection{Distribution with distance}

It can be seen from the events above that the pulses can be found at various distances from the nucleus, in terms of both absolute distance in kilometres and distance relative to the electron cooling boundary. Henri et al. (2017) showed that the relative distance to this boundary organized observations of the diamagnetic cavity well. As the definition of the cooling boundary distance depends on the cometary activity $Q$ we may expect this to bring some order also to the pulse observations.

In order to investigate this statistically, we used ion current data from LAP2 for the full year of 2015. To obtain comparable data, we only use data from two macros with a good distribution over the year, known as 525 and 624, both with LAP2 measuring ion current. The burst-mode data (624) have a sampling frequency of $58.7 \mathrm{~Hz}$, while the normal-mode data (525) are recorded with a frequency of $0.45 \mathrm{~Hz}$. As the typical pulse duration is several tens of seconds, the lower sampling rate is sufficient. For this purpose we have not calibrated the data to MIP densities but used the raw collected LAP current, which is proportional to the plasma density. The data set is similar to what was presented in Eriksson et al. (2017, fig. 6), but the pulse-finding algorithm is slightly refined. The present algorithm uses a threshold for the prominence (the amplitude over a background) of a peak in the LAP2 ion current. Given any signal peak (local maximum), the prominence is defined as the difference of this peak value and the minimum value found between the peak and the nearest point, where the signal reaches at least the same magnitude (or, if it never does, an end point of the 10-min data interval). One such prominence value can be defined before the peak and one after the peak, and the smallest of the two is used. The plot of occurrence statistics as a function of mission date and cometary longitude provided by Eriksson et al. (2017, fig. 6) does not change appreciably by this, but the new method avoids some problems when pulses occur close together. An example of how the pulse detection works is shown in Fig. 3(b), where pulses found by this criterion are marked by purple triangles. The threshold set for the peak prominence is $20 \mathrm{nA}$, which for this event corresponds to a density increase of about $400 \mathrm{~cm}^{-3}$ (Table 2).

We take the number of pulses detected in a non-overlapping 10min interval and plot versus where it was observed. Fig. 7(a) and (b) show pulse counts versus the radial distance $r$ and versus the 


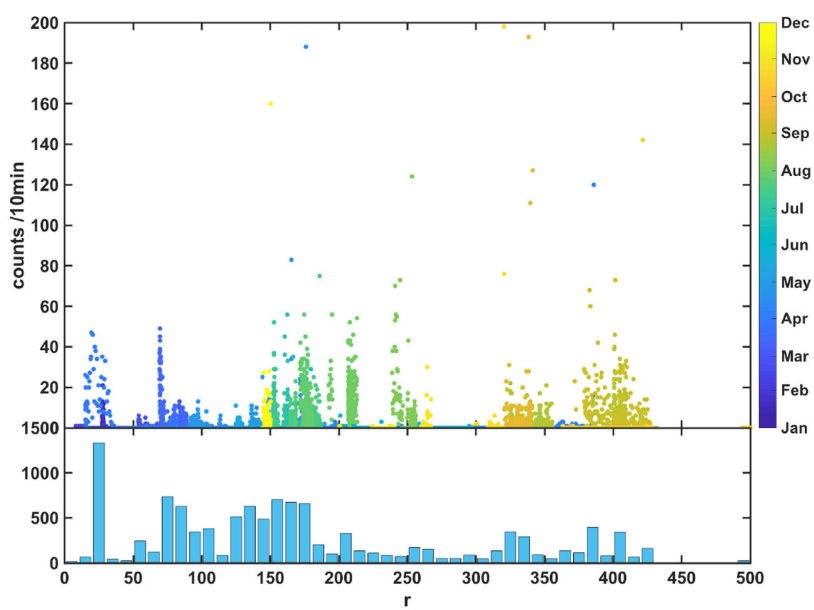

(a)

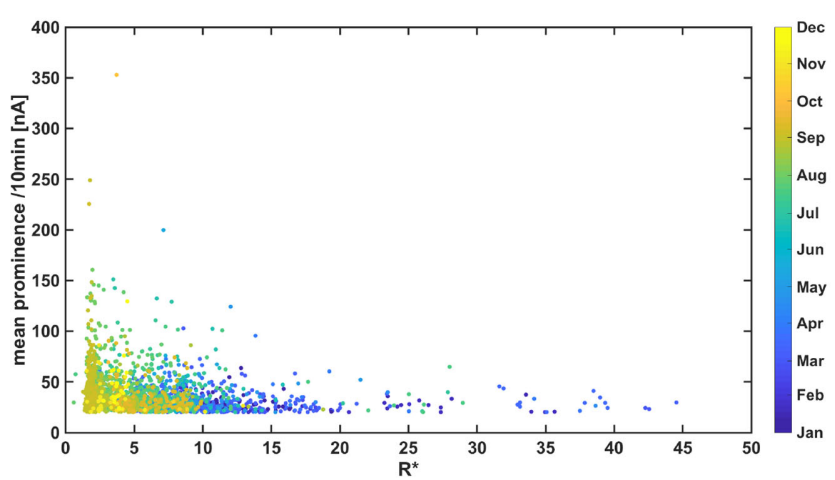

(c)

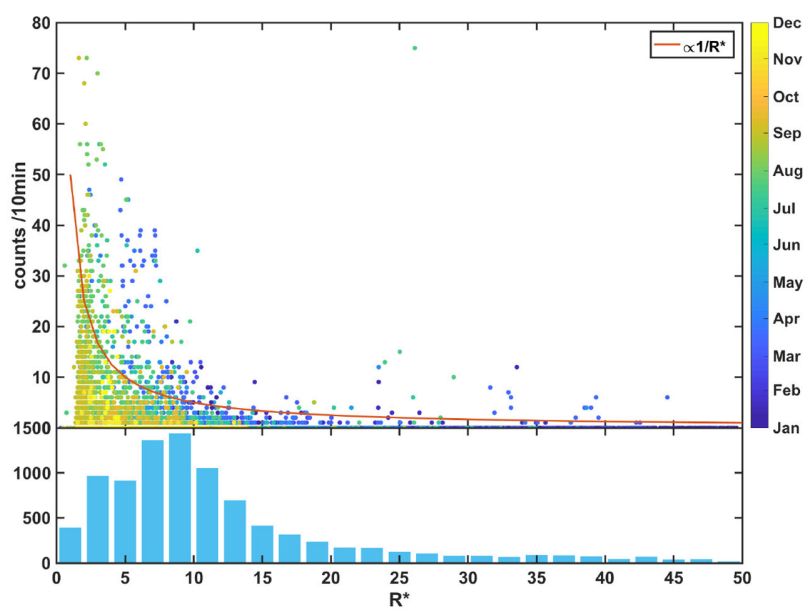

(b)

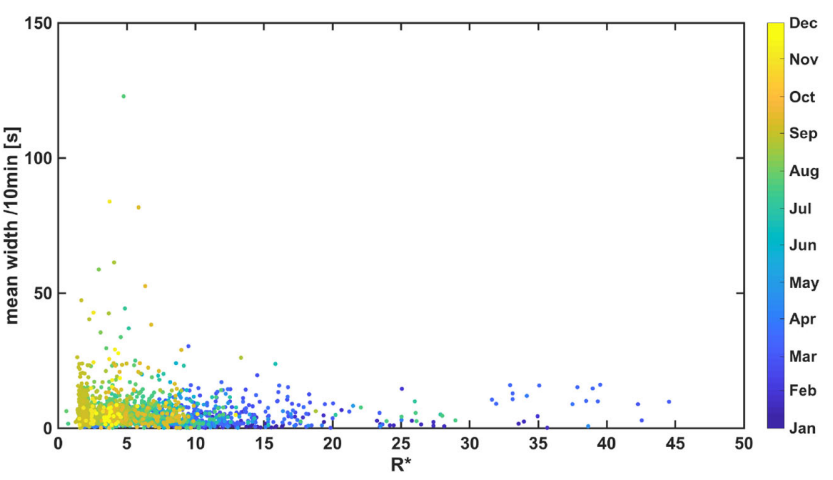

(d)

Figure 7. (a) The number of detected pulses, counts, during a 10-min interval of data from LAP2. This is plotted against the radial distance of Rosetta. No clear correlation is visible. The lower panel shows the number of 10-min intervals spent at a specific radial distance interval. Part (b) is again the number of counts during a 10-min interval, but plotted against the distance of Rosetta with respect to the electron cooling boundary, $R^{*}$. We see that the data are sorted and decay approximately as $1 / R$. The red line gives a $1 / R$ dependence. Most of the data lie where Rosetta is at between 6 and 10 times the cometocentric distance of the exobase. Parts (c) and (d) show the prominence and width of the pulses as sorted by the distance to the exobase.

position of Rosetta with respect to the electron cooling boundary, $R^{*}$, respectively. The histogram below each plot shows the number of data points in a position bin. The number of pulses in a 10-min interval is not well sorted by the radial distance (Fig. 7a). However, from Fig. 7(b) we see that the data are much better organized by position relative to the electron exobase. For comparison, a $1 / R^{*}$ curve is also shown (red line). Rosetta spends most of its time at about 5-10 times the cometocentric distance of the exobase, peaking at 9 , but the higher numbers of pulses are mostly seen closer to the nucleus.

Fig. 7(c) shows the mean prominence of the peaks found in the same 10-min intervals as above. Only plots sorted by $R^{*}$ are shown, as the radial distance $r$ in kilometres did not sort the data well in this case either. As discussed above, the prominence is the amplitude of the pulse compared with the background value around it. This is the LAP probe current rather than the calibrated density, but as the current is proportional to the density we can see that the highest pulses occur closer to the exobase. Fig. 7(d) show the same characteristics for the width in seconds, which is also well sorted by $R^{*}$ but not by $r$ (the latter results not shown). The pulse width varies, but typically lies between a few seconds and a few tens of seconds. Broader pulses were detected mainly when the comet was most active, during
August-December, which is also the period of the events presented above.

\section{DISCUSSION AND CONCLUSION}

\subsection{Summary of observations}

We have presented RPC data for four events that occurred during the months after perihelion. The events show varying radial distance to the nucleus, phase angle and local time. These events all show pulse-like intensifications of plasma density, ion energy and flux, and magnetic field intensity. The enhancement of the density and magnetic field is often very strong, up to an order of magnitude and sometimes even more. The plasma density increase in a pulse is seen in LAP ion and electron currents as well as in MIP plasma frequency data, ICA ion flux and LAP spacecraft potential. The magnetometer time series is smoother than the other data, which can be understood from Biot-Savart's law. The plasma measurements are local, while the magnetic field is an integration of the current density over a large volume.

We could also observe the spacecraft potential becoming more negative within a pulse, but combining the density from the MIP 
plasma frequency with ion and electron currents from LAP shows that the LAP current intensifications are not artefacts of varying spacecraft potential. Instead, the varying spacecraft potential can be interpreted as the varying plasma density. In dense plasmas, however, when the spacecraft potential is sufficiently negative, the response of the LAP electron current to a density intensification is complicated by the spacecraft potential change, and LAP electron current measurements are not always reliable in this situation (Section 3.2). Using the LAP ion current, calibrated to the MIP density from the plasma frequency determination, removes this ambiguity. Pulses are generally seen in both probes (both measuring ion current or one measuring electron current and the other ion current) but they do not look exactly the same, even when the probes are identically operated (see for example Fig. 4). This may be a result of different plasma conditions at the two probes because of the effects of the spacecraft on the plasma, such as the formation of a wake or even a bow wave.

Using the neutral gas number density from COPS, we could define the cometocentric distance of the electron exobase and normalize the cometocentric distance to this. In the events studied here, Rosetta was at 3-10 times the distance of the electron cooling boundary. The full-year statistics showed pulse observations over a larger range of distances, but few are found outside 20 times the height of the exobase. In general, more pulses are found close to the exobase than far away.

The shape of the distribution of pulse observations in Fig. 7(b) is similar to what Henri et al. (2017) found for diamagnetic cavity observations, but the pulses are seen farther out than the cavity. The plasma density in the cavity is much smoother than in the region outside, although density pulses have been found in about 15 percent of all cavity events (Hajra et al. 2018). This is consistent with the fact that we found only a few pulses that are situated inside $2 R^{*}$. In absolute values of the distance normalized to the exobase distance, $R^{*}$, the cavity observations are confined within about $R^{*}<5$, while the pulses we observed are frequently seen at least to $R^{*}=20$ in our statistics. Furthermore, the pulses of highest amplitude and width are found closest to the electron exobase. Our statistics are based on a threshold for how much the magnitude of a current pulse should rise over the background. The fact that we find pulses to have a lower amplitude far away will skew the occurrence statistics in the sense that there may be many but smaller pulses at large distances, where some might be missed owing to the set threshold. While the electron exobase scaling fits well, there could still be some other process scaling in a similar way, for example ion collisionality, which regulates the stability of the cometary ionosphere.

\subsection{Comparison with simulation results}

The observed plasma variations can be compared with the global 3D hybrid simulation model by Koenders et al. (2015), who studied the cometary plasma at an activity of $Q=5 \times 10^{27} \mathrm{~s}^{-1}$. Table 1 shows that this is the relevant range for the events we present. In the hybrid simulation (Koenders et al. 2015, figs 3 and 6), filaments or blobs of high-density plasma were seen to detach from the diamagnetic cavity and move outwards and ultimately tailwards. The density in these simulated pulses seems to reach above the value just inside the cavity by a factor of $2-5$, and sometimes more than an order of magnitude over the density seen adjacent to the pulses. These pulses were seen also in the magnetic field strength, which about doubled in the simulated pulses, although the phasing of the density and magnetic field increases was not always perfect. This corresponds well to what we observed in the events presented in this paper, but there are also differences. The plasma density seen in the simulation just inside the cavity in the plane through the nucleus perpendicular to the interplanetary magnetic field was about $5000 \mathrm{~cm}^{-3}$, while none of our events reaches much more than $1000 \mathrm{~cm}^{-3}$ in the cavity. In addition, the diamagnetic cavity in the simulation extended at most about $50 \mathrm{~km}$ from the nucleus, while in our events as well as in all cavity observations by Goetz et al. (2016a) it is seen to be much bigger. From Koenders et al. (2016), the typical duration of highdensity pulses also seems to be from a few to about $20 \mathrm{~s}$. Similar results are seen in the magnetohydrodynamic models by Rubin et al. (2012). This is mainly comparable to our results, but we also have many examples of wider pulses. It should also be noted that nothing like the 'fingers' of unmagnetized plasma stretching out from the diamagnetic cavity that were inferred by Henri et al. (2017) has been reported from the hybrid simulations. The simulated cavity boundary was not perfectly smooth, but the variations were quite small (Koenders et al. 2015, fig. 6).

The hybrid simulations also suggest that the diamagnetic cavity is most unstable in the plane containing the nucleus and the interplanetary magnetic field (IMF), which we can call the magnetic equatorial plane. We do not have access to the interplanetary magnetic field, because the magnetic field at Rosetta is heavily influenced by mass loading and other cometary processes. Using the local magnetic field, no clear confinement to the magnetic equatorial plane could be found in the events. However, this could be a result of the draping changing the direction of the magnetic field. The pulses could still be more prominent in the real magnetic equatorial plane than elsewhere, because there is clear draping during the months around perihelion (Goetz et al. 2016a).

Using the parameters of the simulation of Koenders et al. (2015), the nominal exobase distance is $20 \mathrm{~km}$. However, the filamentation in the simulation starts at about $50 \mathrm{~km}$, which is at the cavity boundary. Our statistics suggest that the filamentation starts at $R^{*}=1$, which according to Henri et al. (2017) is approximately the position of the cavity boundary for 67P. Our results are therefore consistent with the filamentation starting at the cavity boundary, as seen in the simulations. Note that we use the same definition of the exobase for the simulation and the measured data. So the comparison is meaningful even though the exobase is not a well-defined boundary.

From these points, it appears that the hybrid simulations capture many but not all of the features of our observed pulses, and also some of but not the full physics of the diamagnetic cavity and its surroundings. A clue to the missing physics could be how the distance relative to the electron exobase is observed to organize observations both of the diamagnetic cavity (Henri et al. 2017) and of the pulses outside the cavity (this work). The exobase is the characteristic distance where electron collisions are no longer efficient, and therefore electron kinetic effects become important, which are missing in the hybrid simulations. To fully include such effects, kinetic electrons must be included, as in the particle in cell simulations presented by Deca et al. (2017), which, however, lack collisional processes. Such simulations with a spatial resolution of $10 \mathrm{~km}$ or better will be needed to resolve the diamagnetic cavity and its dynamics, and will when available presumably shed more light on the density and magnetic field pulses presented here.

\subsection{Concluding remarks}

We have shown that the localized density enhancements reported by Eriksson et al. (2017) are common around comet 67P. 
Furthermore, they coincide with enhancement in the magnetic field and ion flux. These characteristics and their distribution in space are at least qualitatively similar to filaments emanating from the diamagnetic cavity in the hybrid simulations by Koenders et al. (2015).

This study leaves some questions unanswered. Among these are the following.

(i) Enhanced ion fluxes up to several hundred electronvolts as reported by Stenberg Wieser et al. (2017) coincide with the density and magnetic field pulses: the acceleration mechanism is unclear.

(ii) The electron temperature in the pulses needs to be investigated, as Eriksson et al. (2017) gives only one example. We saw an indication in Section 3.3.2 that the temperature is different.

(iii) We need to ascertain if there are distinct types of pulses. The detailed examples were taken around perihelion and suggest only one kind of pulse. This could, however, be different at other times. Stenberg Wieser et al. (2017) found five different types of short-lived ion flux enhancements. These types could reflect different types of density structures, for example with or without cold electrons, as reported by Eriksson et al. (2017). Hajra et al. (2018) found that some pulses propagate inside the diamagnetic cavity. This could point to a class of pulses caused by inward-propagating waves reaching the cavity. Our statistics show that fewer pulses are found far out from the nucleus, suggesting that most pulses originate from the inner region. Further study of the cold electrons in the pulses could show the source region of individual pulses, because pulses containing cold electrons must come from the near-nucleus environment, where cooling is efficient.

\section{ACKNOWLEDGEMENTS}

Rosetta is a European Space Agency (ESA) mission with contributions from its member states and the National Aeronautics and Space Administration (NASA). This work has made use of the AMDA and RPC Quick look data base, provided by a collaboration between the Centre de Donnés de la Physique des Plasmas (CDPP) (supported by CNRS, CNES, Observatoire de Paris and Université Paul Sabatier, Toulouse), and Imperial College London (supported by the UK Science and Technology Facilities Council). The research in this paper was funded by the Swedish National Space Board under contracts 171/12 and 109/12. Work at LPC2E/CNRS was supported by ESEP, CNES and by ANR under the financial agreement ANR-15-CE31-0009-01.

\section{REFERENCES}

Balsiger H. et al., 2007, Space Sci. Rev., 128, 745

Bieler A. et al., 2015, A\&A, 7, 1

Broiles T. W. et al., 2016, J. Geophys. Res.: Space Phys., 121, 7407

Carr C. et al., 2007, Space Sci. Rev., 128, 629

de Almeida A. A., Trevisan Sanzovo D., Sanzovo G. C., Boczko R., Miguel Torres R., 2009, Adv. Space Res., 43, 1993

Deca J., Divin A., Henri P., Eriksson A., Markidis S., Olshevsky V., Horányi M., 2017, Phys. Rev. Lett

Edberg N. J. T. et al., 2015, Geophys. Res. Lett., 42, 4263

Edberg N. J. T. et al., 2016, A\&A, 11, 1

Eriksson A. I. et al., 2006, Space Sci. Rev., 128, 729

Eriksson A. I. et al., 2017, A\&A, 605, A15

Gilet N., Henri P., Wattieaux G., Cilibrasi M., Béghin C., 2017,

Glassmeier K.-H., Boehnhardt H., Koschny D., Kührt E., Richter I., 2007, Space Sci. Rev., 128, 1

Goetz C., Volwerk M., Richter I., Glassmeier K.-H., 2016a, MNRAS, 275, 268

Goetz C. et al., 2016b, A\&A, 588, A24

Hajra R. et al., 2018, MNRAS, in press

Hansen K. C. et al., 2016, MNRAS, 462, 491

Hastings D. E., 1995, J. Geophys. Res., 100, 14457

Henri P. et al., 2017, MNRAS, 10, 372

Heritier K. L. et al., 2017, MNRAS, 129, 118

Johansson F. L. et al., 2017, MNRAS, 11, 1

Karlsson T. et al., 2017, Geophys. Res. Lett., 44

Koenders C., Glassmeier K.-H., Richter I., Ranocha H., Motschmann U., 2015, Planet. Space Sci., 105, 101

Koenders C., Perschke C., Goetz C., Richter I., Motschmann U., Glassmeier K. H., 2016, A\&A, 66

Mandt K. E. et al., 2016, MNRAS, 462, 9

Nilsson H. et al., 2007, Space Sci. Rev., 128, 671

Nilsson H. et al., 2015, Science, 347, 1

Nilsson H. et al., 2017, MNRAS, 469, 252

Odelstad E. et al., 2015, Geophys. Res. Lett., 42, 126

Odelstad E., Stenberg-Wieser G., Wieser M., Eriksson A. I., Nilsson H., Johansson F. L., 2017, MNRAS, 469, 568

Richter I. et al., 2015, Ann. Geophys., 33, 1031

Rubin M., Hansen K. C., Combi M. R., Daldorff L. K. S., Gombosi T. I., Tenishev V. M., 2012, J. Geophys. Res.: Space Phys., 117

Stenberg Wieser G. et al., 2017, MNRAS, 469, 522

Taylor M., Altobelli N., Buratti B., Choukron M., 2017, Phil. Trans. A, 1

Tenishev V., Combi M., Davidsson B., 2008, ApJ, 685, 659

Trotignon J. G. et al., 2007, Space Sci. Rev., 128, 713

Tzou C.-y., 2017, PhD thesis, Univ. Bern

Vigren E., Galand M., 2013, ApJ, 772, 33

Vigren E. et al., 2017, MNRAS, 469, 142

This paper has been typeset from a $\mathrm{T}_{\mathrm{E}} \mathrm{X} / \mathrm{L} \mathrm{T} \mathrm{E} \mathrm{X}$ file prepared by the author. 